\title{
Inhibition of cyclo-oxygenase 2 reduces tumor metastasis and inflammatory signaling during blockade of vascular endothelial growth factor
}

\author{
Vascular Cell 3:22 | DOI: 10.1186/2045-824X-3-22 | C Li et al.; licensee Publiverse Online S.R.L. 2011 \\ Received: 13 Apr 2011 | Accepted: 6 Apr 2011 | Published: 6 Apr 2011
}

Fisher Jason $\mathrm{C}^{+} @$, Gander Jeffrey $\mathrm{W}^{+}$, Haley Mary Jo, Hernandez Sonia L, Huang Jianzhong, Chang Yan-Jung,

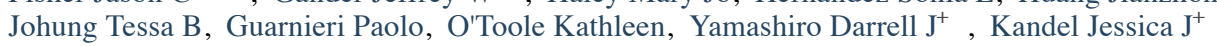

${ }^{+}$Contributed equally ${ }^{@}$ Corresponding author

\begin{abstract}
Vascular endothelial growth factor (VEGF) blockade is an effective therapy for human cancer, yet virtually all neoplasms resume primary tumor growth or metastasize during therapy. Mechanisms of progression have been proposed to include genes that control vascular remodeling and are elicited by hypoperfusion, such as the inducible enzyme cyclooxygenase-2 (COX-2). We have previously shown that COX-2 inhibition by the celecoxib analog SC236 attenuates perivascular stromal cell recruitment and tumor growth. We therefore examined the effect of combined SC236 and VEGF blockade, using the metastasizing orthotopic SKNEP1 model of pediatric cancer. Combined treatment perturbed tumor vessel remodeling and macrophage recruitment, but did not further limit primary tumor growth as compared to VEGF blockade alone. However, combining SC236 and VEGF inhibition significantly reduced the incidence of lung metastasis, suggesting a distinct effect on prometastatic mechanisms. We found that SC236 limited tumor cell viability and migration in vitro, with effects enhanced by hypoxia, but did not change tumor proliferation or matrix metalloproteinase expression in vivo. Gene set expression analysis (GSEA) indicated that the addition of SC236 to VEGF inhibition significantly reduced expression of gene sets linked to macrophage mobilization. Perivascular recruitment of macrophages induced by VEGF blockade was disrupted in tumors treated with combined VEGF- and COX-2-inhibition. Collectively, these findings suggest that during VEGF blockade COX-2 may restrict metastasis by limiting both prometastatic behaviors in individual tumor cells and mobilization of macrophages to the tumor vasculature.
\end{abstract}

\section{Keywords}

COX-2 - angiogenesis - metastasis - VEGF - inflammation - macrophage

\section{Background}

Agents that inhibit vascular endothelial growth factor (VEGF) signaling are increasingly incorporated into treatment regimens for metastatic human cancer, yet the overall benefit of this treatment strategy has been relatively modest [ 1,2]. Both clinical and experimental studies indicate that many or most malignancies will ultimately progress if VEGF blockade is sustained, and that progression may involve both progressive primary tumor growth and enhanced metastasis. The mechanisms for acquired resistance to this treatment approach are thus of great interest, but are still emerging. We previously found that VEGF inhibition significantly reduced primary tumor growth and the incidence of spontaneous lung metastasis in the orthotopic renal SKNEP1 tumor model over a six week treatment period, and regressed established metastases in late-stage tumors [ 3,4$]$. Recent findings, however, indicate that disruption of VEGF signaling and consequent tumor hypoxia may ultimately promote invasion and metastasis in several tumor models [ 5,6], overcoming the initial anti-metastatic effects of limiting angiogenesis. Prior studies suggest that hypoxia-regulated and proinflammatory genes expressed by tumor cells and stroma, such as $C O X-2$, can promote the establishment of metastatic deposits in the lung. For example, Massague and coworkers previously found that $\mathrm{COX}-2$ and other genes involved in vascular remodeling, identified as components in a "lung metastasis gene signature", functioned collectively to promote metastasis in a breast cancer model [ 7,8]. More broadly, much recent data supports a role for systemic inflammation in the promotion of metastasis in general [9], including dissemination to the lung [ 10]. For example, mice genetically prone to autoimmune arthritis are significantly more prone to develop lung metastasis than nonarthritic controls [ 11]. Recruitment of COX-2-expressing macrophages can create an inflammatory proangiogenic environment that strongly promotes tumor growth [12].

Prior work demonstrates that the celecoxib analog SC236 can reduce spontaneous and experimental metastasis, although it is not clear whether this is due to effects on individual tumor cells, on the primary tumor (e.g. angiogenesis), or on the host environment [ 13]. Thus, it is not known whether the addition of SC236 would limit spontaneous lung metastasis in hypoperfused tumors as occurs during VEGF blockade, when primary tumor angiogenesis is restricted but other prometastatic mechanisms may be active.

In previous studies in the SKNEP1 model, we found that treatment with SC236 perturbed tumor angiogenesis and reduced tumor weights [ 14]. SC236 treatment resulted in the formation of erratic, segmentally dilated tumor vessels, marked by a decrease in early pericytes and a marked reduction in differentiated vascular mural cells (VMC). These alterations in vessel structure observed in SC236-treated xenografts differed strikingly from those previously found in this same model during administration of blocking anti-VEGF antibody [ 15]. The potential importance of remodeling is supported by previous work indicating that periendothelial mural cells serve to protect endothelium during VEGF withdrawal [ 16], and by experiments showing that targeting VMC (e.g. by blockade of platelet-derived growth factor B signaling) in combination with VEGF inhibition enhances anti-angiogenesis $[17,18]$. It is not known whether SC236 attenuates acquisition or function of perivascular stromal cells during VEGF inhibition, thus potentially reducing vessel stability. 
In these studies, we used the SKNEP1 model to ask whether COX-2 inhibition might increase the efficacy of VEGF blockade, either by attenuating vessel remodeling, reducing primary tumor blood flow and tumor growth, or by improving control of metastasis. We asked whether restriction of metastasis could be mediated by direct effects of SC236 on SKNEP1 tumor cells, and whether these were altered in hypoxia, by studying tumor proliferation in vivo during SC236 and anti-VEGF treatment, and metabolic and invasive properties of tumor cells in vitro. We asked whether tumor expression of a subset of a lung metastasis signature containing COX-2 [ 7], and more broadly if expression of genes involved in macrophage recruitment, changed during SC236 treatment. Because these mechanisms may collectively contribute to tumor progression in conditions of reduced perfusion, understanding the effects of COX-2 inhibitors during VEGF blockade may hold promise for improving the usefulness of this treatment strategy.

\section{Materials and methods}

\section{Cell line}

The cell line SKNEP-1 (obtained from the American Type Culture Collection, Manassas, VA) was maintained in culture in 75$\mathrm{cm} 2$ flasks with McCoy's 5A medium (Mediatech, Fisher Scientific, Springfield, NJ). Medium was supplemented with $10 \%$ fetal bovine serum and $1 \%$ penicillin/streptomycin (Life Technologies, Grand Island, NY). Cells were grown at $37^{\circ} \mathrm{C}$ in $5 \% \mathrm{CO} 2$ until confluent, harvested, counted with trypan blue staining, washed, and resuspended in sterile PBS (Life Technologies) at a concentration of $10^{7}$ per $\mathrm{mL}$.

\section{Animal model}

The Institutional Animal Care and Use Committee of Columbia University approved all experiments. Four- to 6-week-old female NCR nude mice (National Cancer Institute at Frederick, Frederick, MD) were housed in a barrier facility and acclimated to 12-hour light/dark cycles for at least 24 hours before experimental use.

\section{Tumor implantation}

Mice $(\mathrm{n}=62)$ were anesthetized with intraperitoneal ketamine $(50 \mathrm{mg} / \mathrm{kg})$ and xylazine $(5 \mathrm{mg} / \mathrm{kg})$. The left flank was prepared in a sterile fashion, and an incision was made, exposing the left kidney. An inoculum of $10^{6}$ SKNEP1 tumor cells in $0.1 \mathrm{ml}$ of PBS was injected into the renal parenchyma using a 25-gauge needle. The flank musculature was closed with a single 4-0 Polysorb suture (U.S. Surgical, Norwalk, CT), and the skin incision was closed with staples.

\section{Administration of SC236 and anti-VEGF antibody}

One week after tumor implantation, animals were divided into four cohorts. Treated mice received either (1) SC236 ( $\mathrm{N}=17$; Pfizer, Groton, CT) added to drinking water at a concentration of $30 \mu \mathrm{g} / \mathrm{mL}$ and changed thrice per week as previously described [ 14]; (2) the humanized monoclonal anti-VEGF antibody bevacizumab (N = 17; BV; Genentech, South San Francisco, CA) injected intraperitoneally at $10 \mathrm{mg} / \mathrm{kg}$ biweekly beginning at week 3 ; or (3) combined agents $(\mathrm{N}=15)$. Control mice $(\mathrm{N}=13)$ received standard drinking water and were injected with carrier vehicle on the same schedule. This concentration of SC236 is equivalent to $\sim 6 \mathrm{mg} / \mathrm{kg} / \mathrm{d}$ (anticipated SC236 plasma level $5 \mu \mathrm{g} / \mathrm{ml}$ ). Plasma levels of SC-236 were previously confirmed in our model by high-performance liquid chromatography (HPLC) [14].

\section{Harvesting of specimens and determination of metastases}

At sacrifice, tumors and contralateral kidneys were removed, weighed, and then preserved in $4 \%$ paraformaldehyde for immunohistochemistry. Portions of tumor were flash frozen in liquid nitrogen and stored at $-80^{\circ} \mathrm{C}$. Both lungs were fixed in $10 \%$ formalin for histology. Slides of paraffin-embedded lung tissue were stained by routine $\mathrm{H} \& \mathrm{E}$ methods and examined by a surgical pathologist to determine the presence or absence of metastases, using three levels from each lung.

\section{Immunohistochemistry}

Endothelial cell immunofluorescent staining was done using a rat anti-mouse anti-platelet-endothelial cell adhesion molecule-1 (PECAM-1) monoclonal antibody (MAP0032, Angioproteomie, Boston, MA). Vascular mural cells (VMC) were visualized using a rabbit anti-human $\alpha$-smooth muscle actin ( $\alpha$ SMA) antibody (RB-9010, Lab Vision/Neomarkers, Fremont, CA). Macrophages were visualized using an anti-murine F4/80 antibody (Abcam, AB6640) and an anti-arginase antibody (Santa Cruz Biotech, SC-20150). All microscopy was done using a Nikon Eclipse E600 apparatus.

\section{Quantification of proliferation}

In vivo tumor proliferation ( $\mathrm{N}=3$ for all groups) was examined by immunofluorescent staining for anti-phospho-histone $\mathrm{H} 3$ (Upstate, Inc., Lake Placid, NY), and quantified by computer-assisted image analysis (23-30 fields per group examined).

\section{MMP-2 and -9 zymography}

Tumor extracts ( $\mathrm{N}=3$ for each group) were normalized for protein content, mixed with nonreducing sample buffer, and electrophoresed in $10 \%$ polyacrylamide gels copolymerized with $1 \mathrm{mg} / \mathrm{mL}$ of gelatin. After electrophoresis, gels were washed twice for $15 \mathrm{~min}$ in $2.5 \%$ Triton X-100, rinsed with water, and incubated overnight in activation buffer $(50 \mathrm{mM} / \mathrm{L}$ Tris- $\mathrm{HCl}, \mathrm{pH}$ $7.5,5 \mathrm{mM} / \mathrm{L} \mathrm{CaCl} 2)$ at $37^{\circ} \mathrm{C}$. Digested gelatin bands were visualized by staining with $0.1 \%$ Coomassie blue R-250 in $40 \%$ methanol/20\% acetic acid. Pooled MMPs were used as a positive control (US Biological). Bands were optically scanned for quantification.

\section{MTT Assay}

$2 \times 10^{5}$ SKNEP cells/well were incubated on a 24-well plate (BD Labware, Franklin Lakes, NJ) for 24 hours. Media was then replaced with control media or media with 25 or 50 uM SC-236 (Pfizer, New York, NY) and incubated for 24 or 96 hours at $37^{\circ} \mathrm{C}$ in either normoxia ( $21 \%$ oxygen) or hypoxia ( $2.3 \%$ oxygen). After the incubation period, media was aspirated and replaced 
with serum-free media and MTT labeling reagent (Roche, Indianapolis, IN). After 24 hours, $600 \mathrm{uL}$ isopropanol was added to each well and the resultant solution read in a Life Science UV spectrophotometer (Beckman, Fullerton, CA) at wavelengths of 550 and $690 \mathrm{~nm}$. All samples were plated in triplicate and experiments were repeated 3 times. Assays were repeated with added bevacizumab $(100 \mathrm{ng} / \mathrm{ml})$.

\section{Invasion Assay}

SKNEP cells were serum-starved for 24 hours. $5 \mathrm{uM}$ Cell Tracker Green (Invitrogen) was added to the cells and incubated for 45 $\mathrm{min}$. Media was then aspirated and cells incubated in serum-free McCoy's solution for an additional $45 \mathrm{~min} .8 \times 10^{5}$ cells/well were plated in 24-well, 8 um pore, Matrigel-coated Tumor Invasion System plates (BD Biosciences) with serum-free media containing 0 (control), 25, or 50 uM SC-236. The same media with 15\% FBS was added to the lower well, and the plate incubated for 24 hours in either normoxia or hypoxia (as above). Invading cells were quantified using a Cytofluor series 4000 Fluorescence multi-well plate reader (Applied Biosystems, Foster City, CA) at an excitation wavelength of $485 \mathrm{~nm}$ and an emission wavelength of $530 \mathrm{~nm}$. All samples were plated in triplicate and experiments were repeated 3 times.

\section{Apoptosis}

$10^{5}$ tumor cells per well were plated on a 96-well Microtest Tissue Culture Plate (BD Labware). Either media or media with 25 or $50 \mathrm{uM} \mathrm{SC-236}$ was added, and allowed to incubate in either normoxia or hypoxia for 20 hours. Apoptosis was analyzed using the Cell Death Detection Elisa kit (Roche, Indianapolis, IN). Briefly, media was aspirated, and cells lysed. After a 30 minute incubation period, the solution was centrifuged at $1250 \mathrm{rpm}$ for 10 minutes and the supernatant added to a streptavidin-coated plate. A mixture of anti-histone-biotin and anti-DNA-peroxidase solution was added to each well and mixed for 2 hours. Substrate was then added, and color intensity read using a VersaMax tunable microplate reader (Molecular Devices, Sunnyvale, CA) at a wavelength of $405 \mathrm{~nm}$. Camptothecin was used as a positive control for apoptosis.

\section{Real-time PCR for lung metastasis signature genes}

Total RNA was isolated from xenograft tumors ( $\mathrm{N}=4$ each), using an Ambion ToTally RNA Extraction kit (Ambion, Austin, TX). Real-time PCR was performed using Taqman probes and an ABI 7300 (Applied Biosystems). Normalization of genes of interest was done utilizing the geNorm method described by Vandesompele et al [ 19]. Six human housekeeping genes (ACTB, HMBS, UBC, GAPD, HPRT, PPIA) were used as reference controls.

\section{Microarrays and gene set expression analysis}

HG-U133A GeneChips (Affymetrix, Santa Clara, CA) were used to investigate gene expression in xenografts. cRNA probes were synthesized as recommended by Affymetrix. Briefly, total RNA was isolated in two steps using ToTALLY RNA Total RNA isolation kit (Ambion, Austin, TX) followed by RNeasy (Qiagen, Valencia, CA) purification. Double-stranded cDNA was generated from $5 \mu \mathrm{g}$ of total RNA using a polydT oligonucleotide that contained a T7 RNA polymerase initiation site and the Superscript Choice System kit (Invitrogen, Carlsbad, CA). Biotinylated cRNA was generated by in vitro transcription using the Bio Array High Yield RNA Transcript Labeling System (Enzo, Farmingdale, NY). cRNA was purified using RNeasy and fragmented according to the Affymetrix protocol, and $15 \mu \mathrm{g}$ of biotinylated cRNA hybridized to HGU133A microarrays (Affymetrix). Raw CEL files were processed using Bioconductor packages in an R environment http://www.bioconductor.org) [ 20]. Briefly, quality controls were performed by inspecting Affymetrix ${ }^{\circledR}$ metrics using the simpleaffy package. Probe level signals were then background-corrected, normalized, and summarized using the GC-RMA function. Differential gene expression was computed using an Empirical Bayesian model implemented in the Limma package (Additional File 1Table S1). To determine whether the addition of SC236 broadly changed inflammation-related pathways in BV-treated tumors, we used Gene Set Expression Analysis and GenePattern tools (GSEA, Broad Institute, Cambridge, MA). The MSigDB gene set database $(5,542$ total sets) was queried for gene sets containing inflammation-related pathways. From this, we constructed a 67 gene set matrix. GSEA was used to assess expression of gene sets in this matrix in $\mathrm{BV}$ - and $\mathrm{BV}+\mathrm{SC} 236$-treated samples $(\mathrm{N}=3,4$ respectively). To compute gene enrichment, we permuted by gene (as recommended for small sample sizes) 5000 times. We then computed odds ratios for the genesets identified as being significant by GSEA, defined as ratio of odds for the hits before and after the leading edge. In particular, for each geneset we computed the following:

oddsRatios $=[($ Hits/Misses) BeforeTheLeadingEdge $] /[$ (Hits/Misses) AfterTheLeadingEdge $]$ We used the odds ratios to additionally filter the results, as nominal p-value is not informative in this setting, and the NES has a bias towards bigger genesets.

\section{Enzyme-linked immunosorbent assay (ELISA)}

ELISA for tumor-derived (human) IL4 was performed using the Human IL-4 Quantikine HS ELISA Kit HS400 (R\&D Systems). All assays were performed in duplicate, and then repeated twice. Results of repeated assays were expressed as summary means \pm standard error (square root of interassay variance) [ 21].

\section{Statistical analysis}

Tumor weights were compared by Kruskal-Wallis analysis. The presence or absence of lung metastases was evaluated by Fisher's exact test. Quantitative results of multiply-repeated MTT, invasion, and ELISA assays were assessed using Comprehensive Meta Analysis software (Biostat, Inc., Englewood, NJ). Differences in gene expression by real-time PCR were compared by Student's T-test.

\section{Quantification of changes in vasculature}

Digital images from immunofluorescence and fluorescein-labeled lectin studies were captured from a Nikon E600 fluorescence microscope with a Spot RT slider digital camera (Diagnostic Instruments, Sterling Heights, MI) and stored as TIFF files. Vessel radius was determined by immunofluorescence for $\alpha$ SMA and analyzed using software Adobe Photoshop 7.0 (Adobe Systems, Mountain View, CA) and Image Processing Toolkit (IPTK 5 IPTK 5, Rein deer Graphics, Raleigh, NC). Briefly, background fluorescence is subtracted (AutoLevelDark), Gaussian filter applied to reduce electronic and background noise (Gaussian Blur, radius $=1.0$ pixel), and grayscale levels linearly expanded (Auto Levels). A common threshold level is applied that preserves correct vascular morphology (Threshold). The image is inverted (Invert) and dilated to lessen gaps (Classic Morphology > Dilate), small particles representing background removed (Reject Features), and vessel holes filled in (Fill Holes). The average of the inscribed radius is determined as a measure of vessel radius. 60 images from SK-NEP1-GFP (3 different tumors) and 49 
images from SK-NEP1-Ang1* (3 different tumors) were analyzed. To assess MVD and vessel length, quantitative assessment of tumor vessel architecture was performed by computer-assisted digital image analysis as previously described [ 22]. The fraction of fluorescein-labeled lectin-positive pixels per total field was quantified. Specific changes in vessel architecture are evaluated by quantifying branch points (nodes), end points, and total vessel length. Images are analyzed after application of a common threshold, image inversion, morphological erosion, and skeletonization, again using a combination of Photoshop and Image Processing Toolkit.

\section{Results and Discussion}

The addition of SC236 to VEGF inhibition does not further restrict tumor size, but does significantly reduce the incidence of metastasis as compared to anti-VEGF treatment alone

As compared to controls, SC236 treatment reduced tumor weight by $39 \%$ ( $P=0.042$, Kruskal-Wallis analysis; Figure 1A), whereas tumors treated with the anti-VEGF antibody bevacizumab (BV) were reduced by $76 \%(P=0.0002)$. The addition of SC236 to BV treatment did not further decrease primary tumor weight (74\% reduction from controls, $P=\mathrm{NS}$ as compared to $\mathrm{BV}$ alone). The incidence of lung metastasis was determined by a surgical pathologist blinded as to treatment group, examining 3 levels from the entire right lung (Figure 1B). Nearly all of the control animals had detectable metastases (12/13 mice, 92\%). $65 \%(11 / 17)$ of SC236 treated animals had lung metastases, but this reduction was not statistically significant. The incidence of metastasis was, however, significantly reduced in BV-treated mice to $47 \%$ (8/17 mice), which was significantly different when compared to controls $(P=0.011)$. Strikingly, combining SC236 with BV treatment caused a further reduction in metastasis with only $13 \%(2 / 15)$ of mice having lung metastases. This effect was statistically significant when compared not only to control tumors ( $P=0.0001)$, but also when compared to BV treatment alone $(P=0.046)$. We examined lung metastases histologically using H\&E staining (Additional File 2Figure S1). Metastases in combination-treated mouse lungs appeared smaller than in control or single-agent treated animals, although the low incidence of metastasis in this group (2/15) prevented quantitation of this size reduction. Thus, our data demonstrates that SC236 when combined with VEGF blockade can markedly reduce distant metastasis, although it does not alter primary SKNEP1 tumor size, suggesting a differential effect on this aspect of tumor progression.

Figure 1 


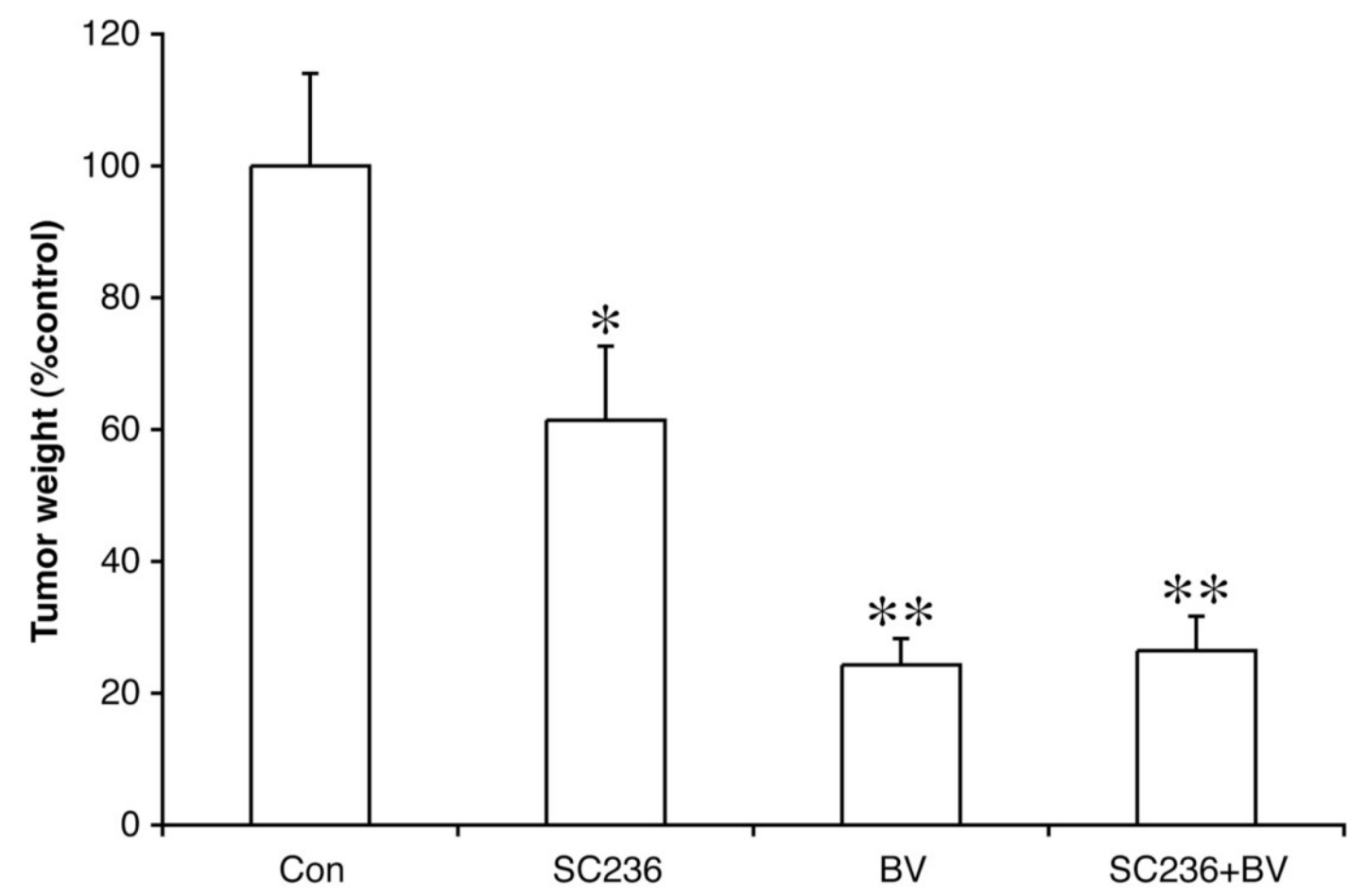

B

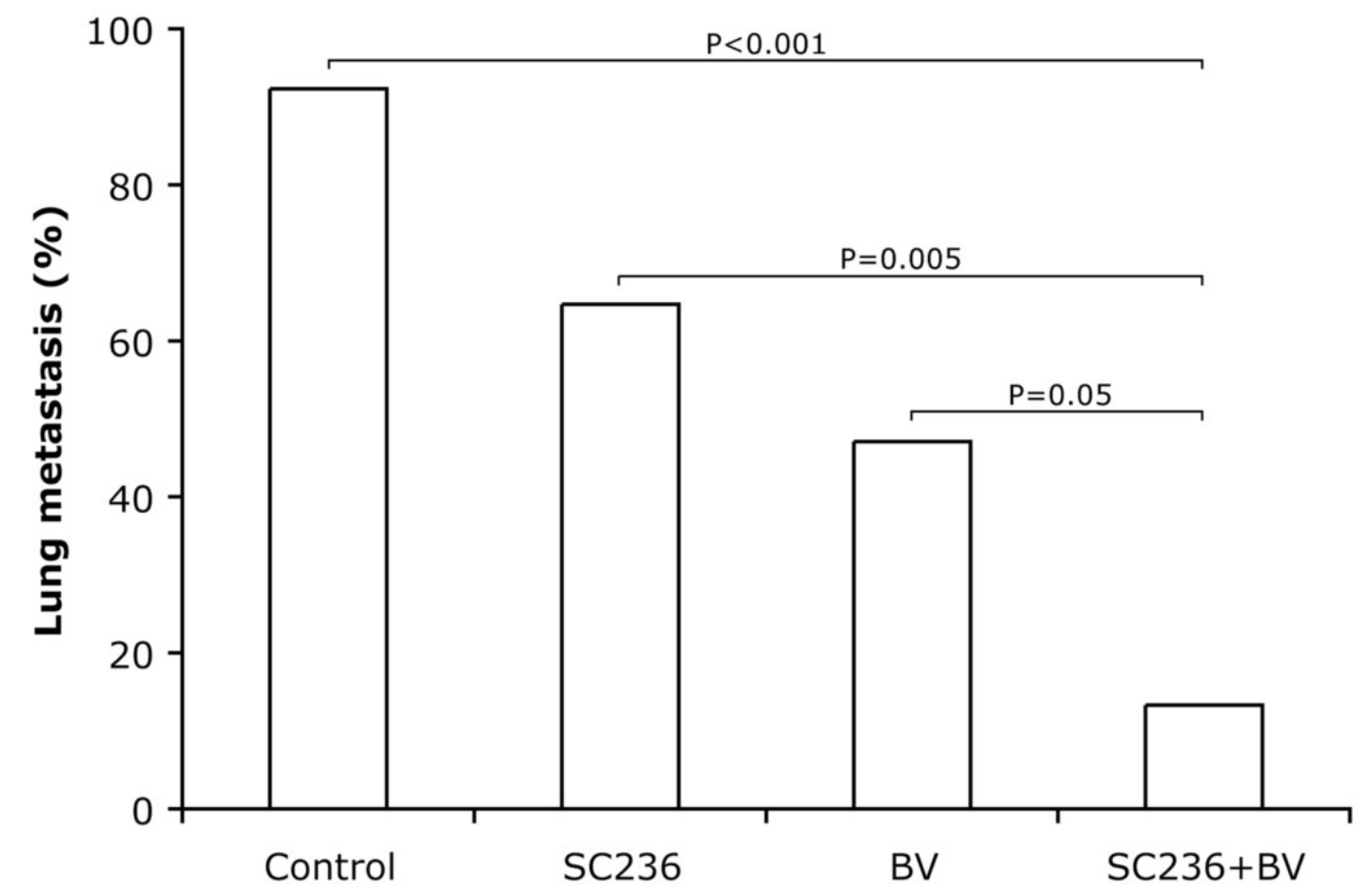

The addition of SC236 to bevacizumab significantly reduces the incidence of metastasis as compared to VEGF inhibition alone. (A)As compared to controls, SC236 treatment reduced tumor weight by $39 \%(P=0.042)$, whereas BV-treated tumors were reduced by $76 \%(P=0.0002)$. The addition of SC236 to BV treatment did not further decrease primary tumor weight (74\% reduction from controls, $P=\mathrm{NS}$ as compared to $\mathrm{BV}$ alone). Tumor weights are expressed as percentage of control tumors, and Kruskal-Wallis analysis used to determine significance. (B)The incidence of metastasis was determined by examining 3 levels from the right lung. 12/13 or $92 \%$ of control animals had detectable metastases, whereas 11/17 (65\%) of SC236 treated animals had lung deposits ( $P=\mathrm{NS}$ ). The incidence of metastasis in BV-treated animals was significantly reduced as compared to controls $(8 / 17$ animals, $47 \%, P=0.011)$. Combining SC236 with BV treatment further significantly reduced metastasis $(2 / 15$ animals, $13 \%, P=0.0001$ as compared to controls and $P=0.046$ as compared to $\mathrm{BV}$ treatment alone). 
The combination of SC236 with VEGF inhibition alters vascular assembly as compared to either treatment alone

To assess changes in the endothelial component of vessels, we performed immunohistochemistry for platelet endothelial cell adhesion molecule-1 (PECAM; Figure 2, left column). Control tumors displayed abundant PECAM(+) neovasculature, with multiple fine branches (arrows). Morphologically, both SC236-treated and BV-treated tumors displayed reduction of branching. In tumors treated with both BV and SC236, surviving endothelial vessels were discontinuous, with irregularly spaced, moderately dilated segments, and near ablation of branches. Mean vascular density was decreased in all treated groups as compared to controls (Figure $2 \mathrm{~B}, \mathrm{p}<0.05$ ). To examine differentiated VMC, we immunostained for $\alpha$-smooth muscle actin ( $\alpha$ SMA, Figure 2 , right column). Quantitation of $\alpha$ SMA immunopositive vasculature demonstrated significant reduction in $\mathrm{BV}+\mathrm{SC} 236$ treated tumors as compared to controls (Figure 2C), potentially consistent with a combined effect on microvessel density and disrupted mural cell coverage of vessels as previously reported [ 14]. Thus, the addition of SC236 to BV treatment may have increased discontinuity in tumor vessel networks, with relative attenuation of VMC recruitment.

Figure 2

A

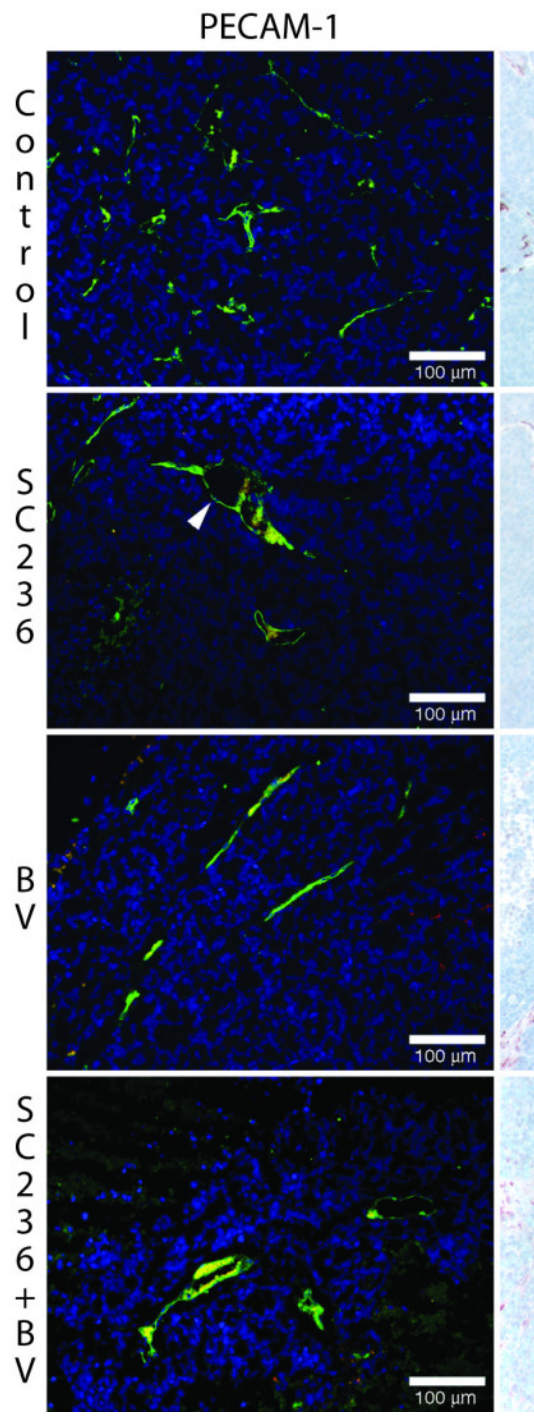

B

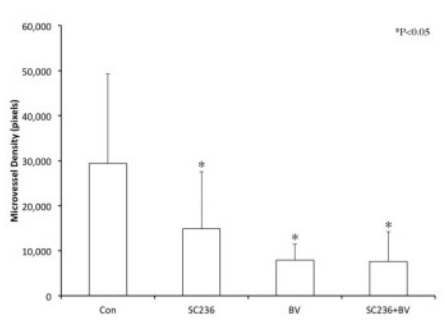

C

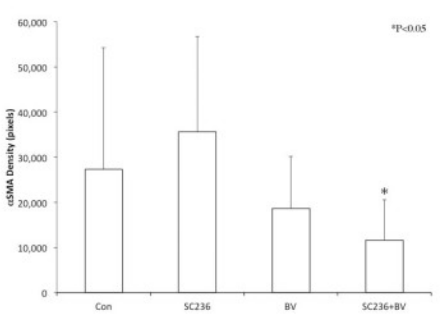

D

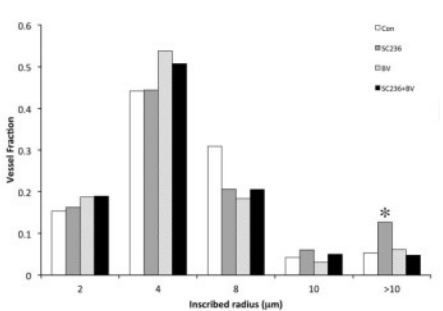

The combination of SC236 with VEGF inhibition alters vascular assembly as compared to either treatment alone. We performed immunohistochemistry for platelet endothelial cell adhesion molecule- 1 to assess changes in endothelial vessels (PECAM; Figure 2, left column). Control tumors displayed abundant PECAM(+) neovasculature, with multiple fine branches (arrows). Morphologically, both SC236-treated and BV-treated tumors displayed reduction of branching. In tumors treated with both BV and SC236, surviving endothelial vessels were discontinuous, with irregularly spaced, moderately dilated segments, and near ablation of branches. Mean vascular density was decreased in all treated groups as compared to controls (Figure 2B). To examine differentiated VMC, we immunostained for $\alpha$-smooth muscle actin ( $\alpha$ SMA, Figure 2 right column). Quantitation of $\alpha$ SMA immunopositive vasculature demonstrated significant reduction only in BV+SC236 treated tumors as compared to controls (Figure 2C), potentially consistent with a combined effect on microvessel density and disrupted mural cell coverage of vessels as previously reported [ 14]. PECAM- and $\alpha$ SMA-immunostaining also suggested that SC236treated xenografts were marked by erratically dilated larger vessels (arrowheads, Figure 2A). To quantify this morphologic change, we measured shifts in distribution of the inscribed radius of mural cell-covered vasculature (Figure 2D, using a previously described method [ 22]). SC236-treated tumor vasculature demonstrated a significant increase in the vessels with inscribed radius $>10 \mu \mathrm{m}$.

PECAM- and $\alpha$ SMA-immunostaining also suggested that SC236-treated xenografts were marked by erratically dilated larger vessels. To quantify this morphologic change, we measured shifts in distribution of the inscribed radius of mural cell-covered vasculature (Figure 2D, using a previously described method [22]). SC236-treated tumor vasculature demonstrated a significant increase in the vessels with inscribed radius $>10 \mu \mathrm{m}$. 
We asked whether SC236 caused changes in metastasis-related tumor cell behaviors under conditions imposed by VEGF blockade, including proliferation, activation of matrix metalloproteases (MMP) -2 and -9 and ability to invade basement membrane. (A)COX-2 is known to modulate tumor cell proliferation, and we previously found that SC236 decreased tumor proliferation in vivo. However, it is not known whe ther this effect would be altered in hypoxia, which might enhance sensitivity to COX-2 or (conversely) stimulate other pathways that promote proliferation. We quantitated phosphohistone $\mathrm{H} 3$ (pH3), using image analysis of immunofluorescent staining ( $\mathrm{N}=3$ and 23-30 sections studied, each condition). SC236 reduced pH3 immunopositive cells in vivo (Figure 3A), as compared to both control and BV-treated tumors (33.8 \pm 3.2 cells/hpf vs. $53.6 \pm$ 3.6 and $46.4 \pm 2.8$ cells/hpf, respectively; $\mathrm{P}<0.005$, both). Combined SC236 and BV treatment also reduced proliferation in comparison to controls $(43.1 \pm 2.4$ cells/hpf, $\mathrm{P}<0.02)$. Thus, SC236 restriction of proliferation persisted during VEGF blockade, but was not significantly altered. (B)Activation of matrix metalloproteinases has been linked to metastasis of primary tumors to the lung [ 23, 24], and can be modulated by VEGF and COX-2 signaling [ 25, 26]. Using gelatin zymography, we examined MMP-2 and MMP-9 in tumor samples, and quantified activity by comparing optical band density in each treatment group (Figure 3B , N = 3 each). MMP-2 activity was unchanged in each condition (not shown). MMP-9 activity was significantly reduced in each treatment group as compared to controls (versus SC236 group, 35\%; in BV, 34\%, BV+SC36, 25\%; $\mathrm{P}<0.03$, each). Thus, combined SC236 and BV did not further reduce MMP9 activity. (C)We used the MTT assay to determine whether hypoxia, SC236 concentration, or duration of exposure would reduce viability in SKNEP1 tumor cells. We have previously reported that $30 \mu \mathrm{g} / \mathrm{mL} \mathrm{SC} 236$ in drinking water results in serum concentrations between 25 and $50 \mu \mathrm{M} / \mathrm{mL}$ [ 14]; therefore, we tested effects at these two concentration levels. We found that both hypoxia and increasing concentration suppressed activity measured by MTT, with this effect enhanced at increased duration $(96 \mathrm{~h})$. For example, at $24 \mathrm{~h}$ significant reduction as compared to controls $(9.9 \%, \mathrm{P}<0.001)$ was found only at the higher concentration $(50 \mu \mathrm{M})$ and in hypoxia, whereas at $96 \mathrm{~h}$ significant reduction was found in normoxia at the $50 \mu \mathrm{M}$ concentration (8\%), and in hypoxia at both 25 and $50 \mathrm{uM} \mathrm{(16 \%} \mathrm{and} 44 \%$, respectively; $\mathrm{P}<0.001$, all). These results support an increased effect of SC236 in hypoxic conditions, as may occur in tumor regions during VEGF blockade. We repeated these studies in the presence of $\mathrm{BV}$, and found no effect. (D)We assessed the ability of SC236 to restrict tumor cell invasion through basement membrane (Matrigel), since intravasation is a key step in metastasis. Invasion was significantly reduced only at the $50 \mu \mathrm{M}$ concentration level, by $7 \%$ in normoxia and $14 \%$ in hypoxia $(\mathrm{P}<0.005$, each), consistent with a modest restriction by SC236. In summary, SC236 reduces metastatic behaviors in tumor cells: proliferation in vivo and viability and migration through basement membrane in vitro, with the latter two effects enhanced by hypoxia.

Figure 3

A

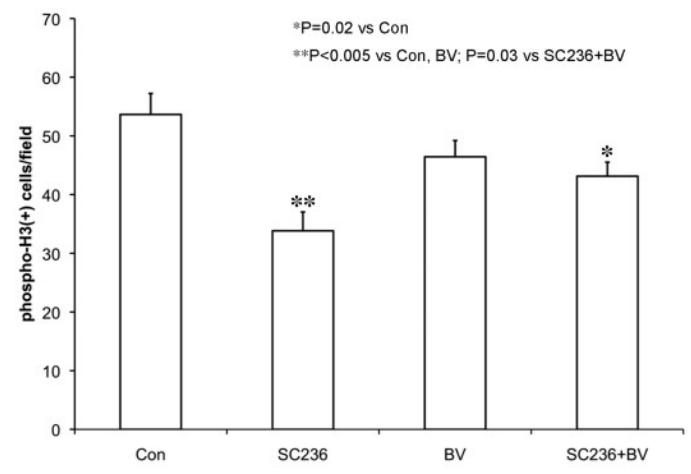

C

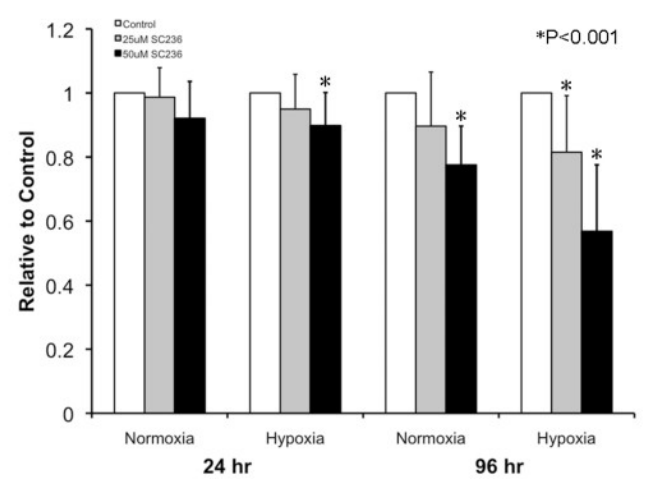

B

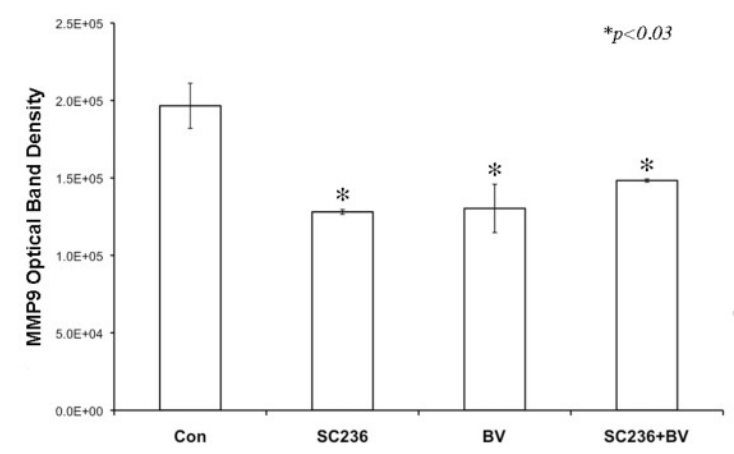

D

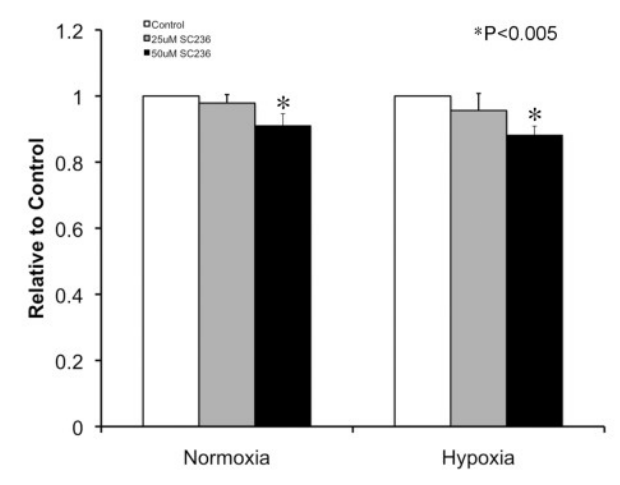

SC236 affects proliferation and invasive properties of SKNEP1 tumors in vivo and in vitro . (A)Quantification of phosphohistone $\mathrm{H} 3(\mathrm{pH} 3)(\mathrm{N}=3 ; 23-30$ sections studied, each condition) demonstrated that SC236 reduced proliferation in vivo as compared to both control and BV-treated tumors $(33.8 \pm 3.2$ cells $/ \mathrm{hpf}$ vs. $53.6 \pm 3.6$ and $46.4 \pm 2.8 \mathrm{cells} / \mathrm{hpf}$, respectively; $\mathrm{P}<0.005$, both). Combined $\mathrm{SC} 236+\mathrm{BV}$ treatment also reduced proliferation versus controls $(43.1 \pm 2.4$ cells/hpf, $\mathrm{P}<0.02$ ). Thus, SC236 restriction of proliferation persisted during VEGF blockade, but was not altered. (B)We assessed MMP-2 and MMP-9 activation, which can promote lung metastasis and interacts with VEGF and COX-2 status, using gelatin zymography [ 23-26] in each treatment group ( $\mathrm{N}=3$ each). MMP-2 activity was unchanged (not shown). MMP-9 activity was significantly reduced in each treatment group as compared to controls (versus SC236 group, 35\%; in BV, 34\%, SC36+BV, 25\%; P <0.03, each). Thus, combined SC236 and BV did not further reduce MMP9 activity. (C)We used the MTT assay to determine whether hypoxia, SC236 concentration, or duration of exposure would reduce SKNEP1 tumor cell viability. Both hypoxia and increasing concentration suppressed activity measured by MTT, with effects enhanced by increasing duration $(96 \mathrm{~h})$. These results support an increased effect of SC236 in hypoxic conditions, as may occur during 


\section{SC236 does not increase apoptosis in SKNEP1 cells in normoxia or hypoxia}

COX-2 signaling can interfere with apoptotic mechanisms in some cell types and in some settings. Therefore, we examined the effect of SC236 on SKNEP1 cell apoptosis in vitro. No increase in apoptosis was found at either concentration of SC236, and no effect of hypoxia was detected (not shown). Thus, increasing apoptosis may not contribute to restriction of metastasis in this system.

The addition of SC236 does not alter expression of lung metastasis signature genes during VEGF blockade

We detected expression of 7 genes (COL6A1, CSF2RA, CXCR4, KRT81, MATN2, SPARC, TNC) included in the lung metastasis gene signature described by Minn et al. [ 8] in tumor extracts from each group ( $\mathrm{N}=6$, controls, SC236-treated, BVtreated; $\mathrm{N}=5, \mathrm{BV}+\mathrm{SC} 236$ treated; Additional File 3Figure S2). Comparison was performed by real-time PCR. No significant differences between BV and SC236+BV treated tumors were found, suggesting that SC236 did not reduce lung metastasis by suppressing expression of these genes.

The addition of SC236 alters expression of gene sets representing macrophage-related inflammatory pathways in BV-treated tumors

COX-2 activity broadly influences inflammation, which appear increasingly implicated in tumor development, progression, and metastasis [9]. It is possible that SC236 improves control of metastasis in BV-treated tumors in our model by reducing expression of inflammation-related genes that act in concert. We therefore used gene set expression analysis (GSEA) and a matrix of 67 gene sets representing pathways linked to macrophage recruitment selected from the Molecular Signatures Database (MSigDB, Broad Institute, Cambridge, MA) to explore this possibility in Affymetrix HGU133A microarray datasets from xenografts $(\mathrm{N}=3,4$ respectively, for $\mathrm{BV}$, and $\mathrm{BV}+\mathrm{SC} 236$ groups). 20 gene sets were significantly negatively enriched (repressed) in combination in SC236+BV treated as compared to BV-treated tumors (Table 1). No positively enriched gene set had an odds ratio $>2.0$, whereas the top 5 negatively enriched gene sets were significant both by GSEA normalized enrichment score and had odds ratios $>2$. Leading edge analysis of these sets suggested involvement of cytokines, with interleukin-4 (IL40 the most significantly suppressed gene in the leading edge analysis. IL4 plays a key role in recruiting and activating tumorassociated macrophages [ 27] and can be suppressed by COX-2 inhibition in inflammatory conditions [ 28]. Therefore, we examined expression of IL4 protein by ELISA in BV-treated versus SC236+BV-treated tumors, and found a $60 \%$ reduction in combined-treatment tumors $(3.1 \pm 1.7 \mathrm{pg} / \mathrm{ml}, \mathrm{BV}$ treated; $1.2 \pm 0.3 \mathrm{pg} / \mathrm{ml}, \mathrm{SC} 236+\mathrm{BV}$-treated, $P=0.056)$.

Table 1

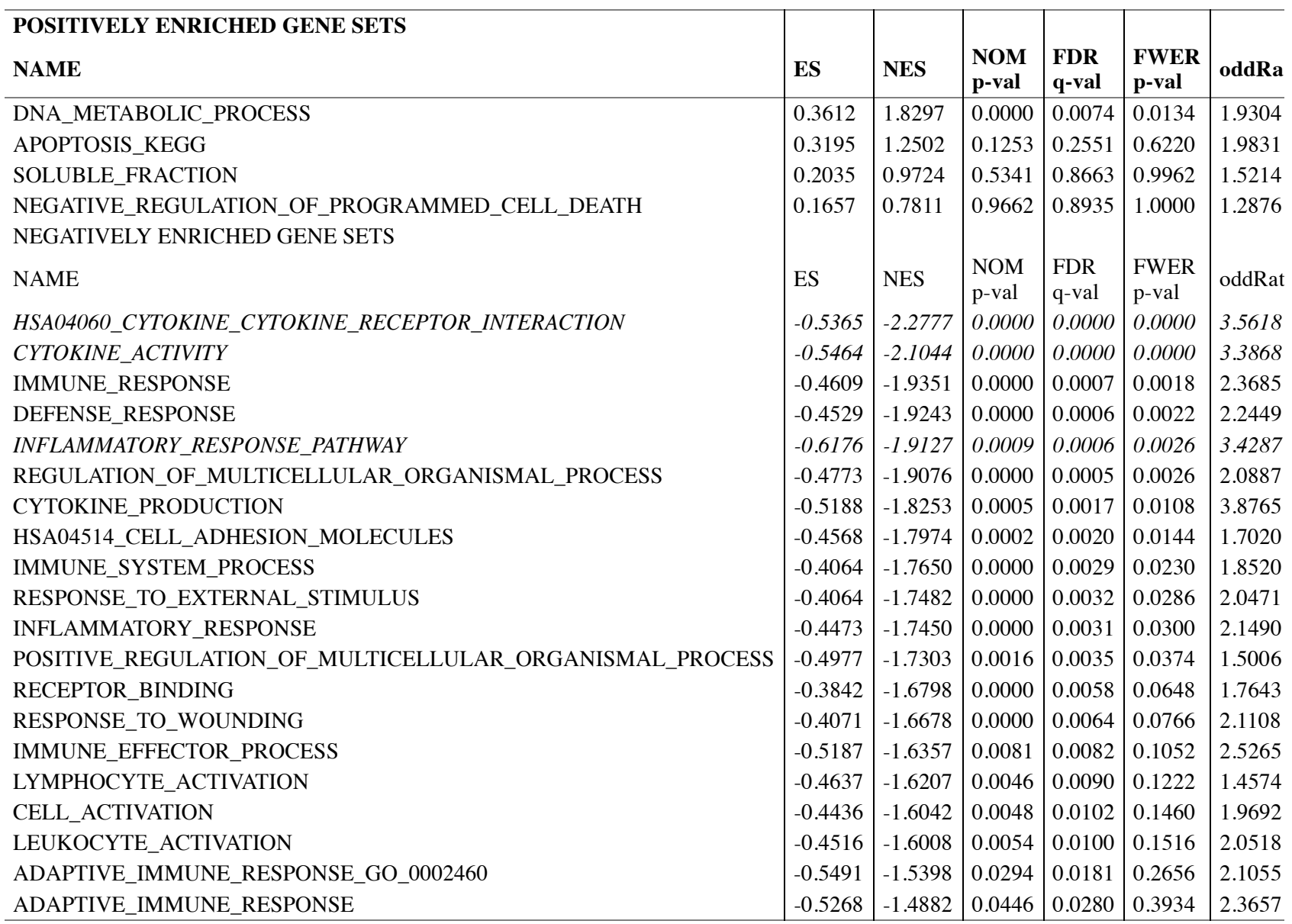

To assess the possibility that SC236 improves control of metastasis in the context of VEGF blockade by broadly reducing expression of inflammation-related genes, we used gene set expression analysis (GSEA) and a matrix of 67 gene sets representing pathways linked to macrophage recruitment selected from the Molecular Signatures Database (MSigDB, Broad Institute, Cambridge, MA) to explore this possibi in Affymetrix HGU133A microarray datasets from xenografts ( $\mathrm{N}=3$, 4 respectively, for BV, and BV+SC236 groups). 20 gene sets were significantly negatively enriched (repressed) in combination in SC236+BV treated as compared to BV-treated tumors; no positively enriched set had an odds ratio $>2.0$ (Table 1). Among the negatively enriched sets, we selected those with odds ratios $>3.0$ in the top 5 for further 
analysis. Thus, it is possible that, in the context of VEGF inhibition, SC236 acts in part by limiting the ability of tumors to promote recruitme of macrophages, key elements in an inflammatory state that facilitates metastasis.

The addition of SC236 alters expression of gene sets associated with macrophage recruitment pathways in BV-treated tumors

\section{Macrophage recruitment is altered by $\mathrm{BV}$ and SC236 treatment}

These results led us to examine distribution of macrophages in treated tumors, using the mouse macrophage marker F4/80 (Figure 4A). Morphologically, there appeared to be increased irregularity of the perivascular F4/80 signal in the combinedtreatment group. Quantification of F4/80 immunopositivity demonstrated significant increase in BV and SC236+BV treated groups as compared to controls (Figure 4B ). To detect shifts in distribution of polarized macrophages, we performed immunohistochemistry for the M1 marker TNF $\alpha$ (Figure 4C). TNF $\alpha$-immunopositive cell distribution appeared similar to the patterns for F4/80 signal. Immunohistochemistry for the M2 marker arginase demonstrated immunopositive cells primarily in necrotic areas of tumors; quantification of arginase signal did not change between treatment groups (data not shown).

\section{Figure 4}

A

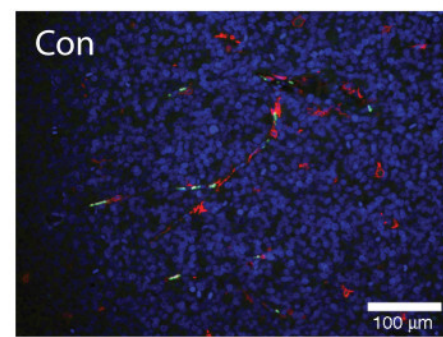

$\mathrm{F} 4 / 80$
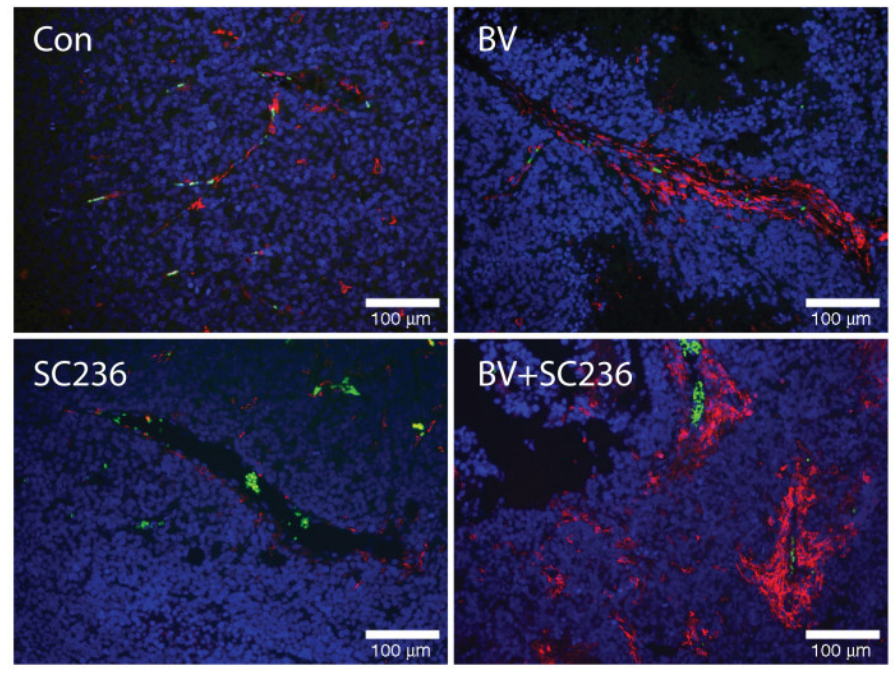

B

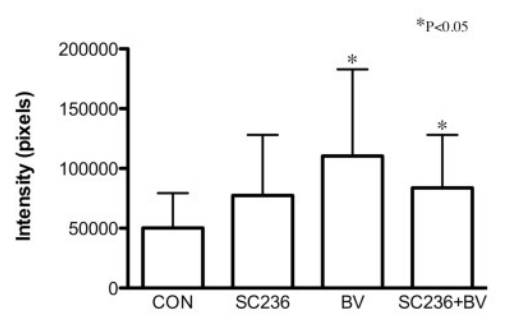

\section{TNF $\alpha$}
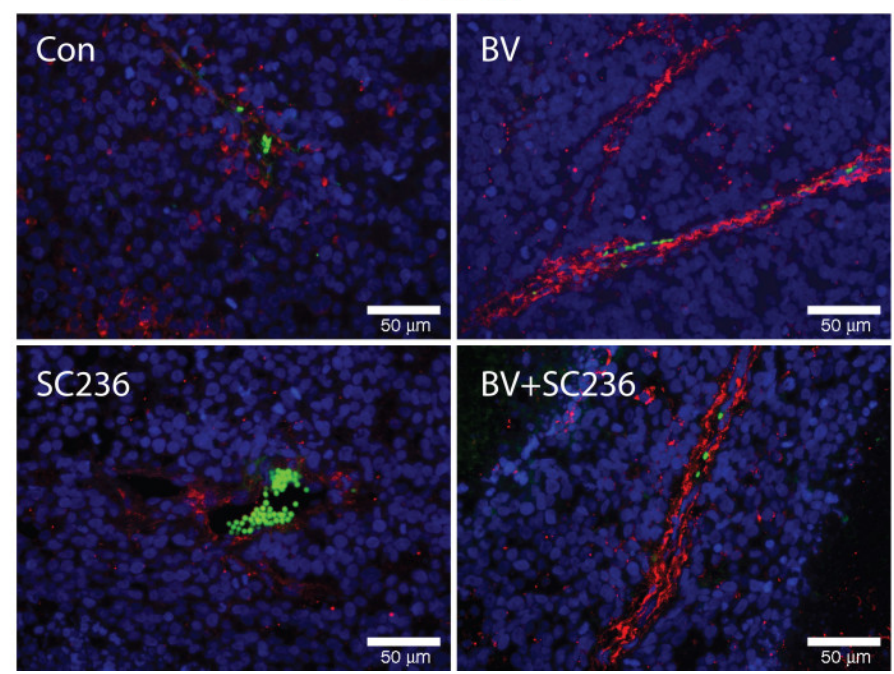

Macrophage recruitment is altered by BV and SC236 treatment. Distribution of macrophages was examined using the mouse macrophage marker F4/80 (Figure 4A). Morphologically, F4/80 appeared to be increased in BV- and BV+SC236-treated tumors, with somewhat more irregularity in the latter. Quantified F4/80(+) signal was significantly increased in BV- and $\mathrm{SC} 236+\mathrm{BV}$-treated groups versus controls (Figure 4B). TNF $\alpha$-immunopositive cell distribution appeared similar to the patterns for F4/80 signal (Figure 4C). The M2 marker arginase was detected in cells primarily in necrotic regions, which did not change with treatment (not shown).

\section{Conclusions}

While inhibition of VEGF signaling has become an established cancer therapy, it has become evident that its overall impact is relatively modest, with virtually all patients developing progressive disease $[5,6]$. One proposed mechanism for the limitations of this approach is the alteration in tumor gene expression induced by hypoperfusion. Because it is regulated by hypoxia, a consequence of treatments that restrict blood supply, COX-2 may participate in this compensatory response to VEGF inhibitors. In addition, COX-2 signaling is implicated in multiple processes that contribute to tumor progression, including proliferation, 
survival, angiogenesis, and invasion. In these studies, we asked whether inhibition of COX-2 by SC236 would enhance the effects of VEGF blockade in the SKNEP1 model, either by perturbing compensatory vascular remodeling and primary tumor perfusion, or by further reducing the incidence of lung metastasis.

SC236 treatment significantly restricted primary tumor weight as compared to controls, as did BV. However, the addition of $\mathrm{SC} 236$ to BV treatment did not result in any further reduction, leading us to ask how this combination affected tumor vasculature and consequent tumor viability. As in our prior studies, both agents impaired angiogenesis, with reduction in branching and pruning of smaller vessels [ 14]. SC236 disrupted vascular assembly, with erratic dilatation of large vessels. Tumors treated with combined agents displayed elements of these two patterns, with preserved VMC coverage of surviving vessels but irregular segmental dilatation and discontinuity. Tumor proliferation was not further reduced in vivo from treatment with SC236 alone. Thus, in our model the ability of SC236 to perturb VMC recruitment did not sufficiently destabilize vasculature during VEGF inhibition to further limit tumor growth.

In the SKNEP1 model, inhibition of VEGF reduces the incidence of lung metastasis, and can cause initial reg ression of established metastases in later-stage tumors [ 4]. However, SKNEP1 tumors [ 29] and metastases (our unpublished observation) recur if VEGF inhibition is prolonged. Thus, our system may serve as an example of a tumor type in which metastasis is initially sensitive to VEGF blockade, in contrast to others in which it may actually be increased [ 5,6]. The addition of SC236 to BV treatment did significantly improve control of metastasis, by reducing incidence. This finding suggests that SC236 may have limited one or more tumor cell behaviors that specifically contribute to establishment of metastasis, such as the ability to transit tumor parenchyma, invade vasculature, or survive as implants in the lung, distinct from effects on primary tumor growth. Consistent with this concept, proliferation in primary tumors was not further restricted in BV+SC236- as compared to SC236treated xenografts. However, we found that viability of SKNEP1 cells (as assessed by MTT) tended to be restricted by SC236 in vitro in hypoxia, particularly if exposure were prolonged or at a higher concentration. It is possible that proliferation is supported in a primary tumor in vivo by secreted products of adjacent nonhypoxic regions, since BV+SC236 did not completely devascularize xenografts, or by host cells that contribute to tumor stroma. Invasion of tumor cells into basement membrane in vitro was similarly restricted by $50 \mu \mathrm{M} \mathrm{SC} 236$, with possible enhancement of this effect by hypoxia. However, while MMP-9 activity was reduced in both SC236- and BV-treated tumors, there was no further reduction in activity in BV+SC236-treated xenografts, suggesting that decreased invasion may not be mediated by diminished MMP-9. No effect of SC236 on tumor cell apoptosis was detected. Taken together, these findings are consistent with an effect on prometastatic behaviors (viability, invasion of vascular basement membrane) in individual tumor cells.

Because alteration in COX-2 signaling may affect tumor cell expression of other genes that specifically promote lung metastasis, we asked whether SC236 treatment altered expression of a subset of a previously described lung metastasis gene signature that includes COX-2 [ 7,8]. While expression of these genes was detected in our model system, the addition of SC236 to BV did not reduce expression as compared to $\mathrm{BV}$-treated tumors. More broadly, mobilization of macrophages by an inflammatory tumor microenvironment can also contribute to metastasis [9-11]. Given the ability of SC236 to block COX-2 activity, we asked whether the addition of SC236 to BV treatment limited primary tumor expression of gene sets implicated in macrophage-related pathways. Using gene set expression analysis, we found a shift toward significant suppression of gene sets containing cytokines that mediate such responses. In particular, we detected reduced expression of IL4 mRNA and protein. IL4 plays an established role in activating tumor-associated macrophages, and can be regulated by COX-2 activity. Suppression of this cytokine by COX2 inhibition in the context of VEGF blockade might therefore contribute to attenuating a prometastatic tumor microenvironment. BV treatment quantitatively increased perivascular macrophage recruitment. The addition of SC236 did not limit this increase, or modulate distribution of cells bearing M1 or M2 markers. Thus, the effect of SC236 on tumor-associated macrophages in the context of VEGF blockade does not appear to include quantitative changes in recruitment.

Thus, our data provides evidence that inhibition of COX-2 may limit metastasis in the setting of VEGF inhibition, distinct from its effect on primary tumor angiogenesis and growth. Restriction of metastasis may result from reduced viability or ability to transit basement membrane in individual tumor cells. In addition, diminished primary tumor angiogenesis may have limited tumor cell access to the circulation, limiting hematogenous dissemination. We detected increased perivascular recruitment of macrophages in BV-treated tumors, and shifts in gene expression related to macrophage function between BV- and BV+SC236treated tumors. However, we did not detect histologic changes in the distribution of macrophages when SC236 treatment was added to BV. Further investigation of functional effects of COX-2 blockade on interaction of tumor cells and stroma may assist in better understanding of these alterations, and in optimizing the use of anti-VEGF treatments in cancer patients at risk for metastatic progression.

Additional file 1: Table S1. LimmaAnnotated.xls: Differential gene expression was computed using an Empirical Bayesian model implemented in the Limma package. Briefly, we analyzed microarray data obtained using HGU133A GeneChips (Affymetrix, Santa Clara, CA) to investigate gene expression in xenografts. Raw CEL files were processed using Bioconductor packages in an R environment http://www.bioconductor.org). Quality controls were performed by inspecting Affymetrix ${ }^{\circledR}$ metrics using the simpleaffy package. Probe level signals were then background-corrected, normalized, and summarized using the GC-RMA function, and differential gene expression then computed as above. (XLS $8 \mathrm{MB}$ )

Additional file 2: Figure S1. Metastases in combination SC236 and BV-treated mouse lungs appeared smaller than in control or single-agent treated animals. The low incidence of metastasis in this group $(2 / 15)$ prevented quantitation of this size reduction, although it may reflect decreased efficiency of metastasis in this group. (PDF $421 \mathrm{~KB}$ )

Additional file 3: Figure S2. The addition of SC236 does not alter expression of lung metastasis signature genes during VEGF blockade. We examined expression of 7 genes (COL6A1, CSF2RA, CXCR4, KRT81, MATN2, SPARC, TNC) included in the lung metastasis gene signature described by Minn et al. (8) in tumor extracts from each group ( $\mathrm{N}=6$, controls, $\mathrm{SC} 236$-treated, $\mathrm{BV}$-treated; $\mathrm{N}=5, \mathrm{BV}+\mathrm{SC} 236$ treated). Comparison was performed by real-time PCR. No significant differences between BV and SC236+BV treated tumors were found, suggesting that SC236 did not reduce lung metastasis by suppressing expression of these genes. (PDF $266 \mathrm{~KB}$ )

\section{Declarations}


These studies were supported by the National Institutes of Health (1R01CA124644, to DY, 1R01 CA100451 to JK), the Pediatric Cancer Foundation (to DY and JK), and the Sorkin Fund (to JK). Lucy Eljuga provided technical assistance.

\section{Authors' original submitted files for images}

Below are the links to the authors' original submitted files for images.

Authors' original file for figure 1

Authors' original file for figure 2

Authors' original file for figure 3

Authors' original file for figure 4

Jason C Fisher, Jeffrey W Gander, Darrell J Yamashiro and Jessica J Kandel contributed equally to this work.

\section{Competing interests statement}

The authors declare that they have no competing interests.

\section{Authors' contributions}

JCF contributed to design, and planned and executed mouse experiments, tissue analyses, gel chromatography, and migration studies. MJH, SLH, and JH performed mouse tumor modeling, immunohistochemistry, and tissue analyses. YJC performed ELISA studies. TBJ performed macrophage studies. PG analyzed raw microarray data. KO analyzed mouse lungs for metastasis. DJY and JJK conceived of the study, supervised its execution, and wrote the manuscript.

\section{References}

1. Ebos JM Lee CR Kerbel RS Tumor and host-mediated pathways of resistance and disease progression in response to antiangiogenic therapy Clin Cancer Res 200915502050252743513 10.1158/1078-0432.CCR-090095

2. Gaur P, Bose D, Samuel S, Ellis LM. Targeting tumor angiogenesis. Semin Oncol. 2009;36:S12-19. View Article Google Scholar

3. Rowe DH, Huang J, Kayton ML, Thompson R, Troxel A, O'Toole KM, Yamashiro D, Stolar CJ, Kandel JJ. Anti-VEGF antibody suppresses primary tumor growth and metastasis in an experimental model of Wilms' tumor. J Pediatr Surg. 2000;35:30-32; discussion 32-33.

View Article Google Scholar

4. Huang J Frischer JS Serur A Kadenhe A Yokoi A McCrudden KW New T O'Toole K Zabski S Rudge JS Regression of established tumors and metastases by potent vascular endothelial growth factor blockade Proc Natl Acad Sci USA 200310077857790164665 10.1073/pnas.1432908100

5. Ebos JM Lee CR Cruz-Munoz W Bjarnason GA Christensen JG Kerbel RS Accelerated metastasis after shortterm treatment with a potent inhibitor of tumor angiogenesis Cancer Cell 2009152322394540346 10.1016/j.ccr.2009.01.021

6. Paez-Ribes M Allen E Hudock J Takeda T Okuyama H Vinals F Inoue M Bergers G Hanahan D Casanovas O Antiangiogenic therapy elicits malignant progression of tumors to increased local invasion and distant metastasis Cancer Cell 2009152202312874829 10.1016/j.ccr.2009.01.027

7. Minn AJ Kang Y Serganova I Gupta GP Giri DD Doubrovin M Ponomarev V Gerald WL Blasberg R Massague J Distinct organ-specific metastatic potential of individual breast cancer cells and primary tumors J Clin Invest 20051154455539194 10.1172/JCI22320

8. Minn AJ Gupta GP Padua D Bos P Nguyen DX Nuyten D Kreike B Zhang Y Wang Y Ishwaran H Lung metastasis genes couple breast tumor size and metastatic spread Proc Natl Acad Sci USA 20071046740 $6745187185610.1073 /$ pnas.0701138104

9. Mantovani A. Cancer: Inflaming metastasis. Nature. 2009;457:36-37. View Article Google Scholar

10. Hiratsuka S, Watanabe A, Sakurai Y, Akashi-Takamura S, Ishibashi S, Miyake K, Shibuya M, Akira S, Aburatani H, Maru Y. The S100A8-serum amyloid A3-TLR4 paracrine cascade establishes a pre-metastatic phase. Nat Cell Biol. 2008;10:1349-1355.

View Article Google Scholar

11. Roy LD Pathangey LB Tinder TL Schettini JL Gruber HE Mukherjee P Breast cancer-associated metastasis is significantly increased in a model of autoimmune arthritis Breast Cancer Res 200911 R562750117 $10.1186 / \mathrm{bcr} 2345$

12. Nakao S Kuwano T Tsutsumi-Miyahara C Ueda S Kimura YN Hamano S Sonoda KH Saijo Y Nukiwa T Strieter RM Infiltration of COX-2-expressing macrophages is a prerequisite for IL-1 beta-induced neovascularization and tumor growth J Clin Invest 2005115297929911257532 10.1172/JCI23298 
13. Roche-Nagle G Connolly EM Eng M Bouchier-Hayes DJ Harmey JH Antimetastatic activity of a cyclooxygenase-2 inhibitor Br J Cancer 2004913593652409822

14. Lee A, Frischer J, Serur A, Huang J, Bae JO, Kornfield ZN, Eljuga L, Shawber CJ, Feirt N, Mansukhani M, et al. Inhibition of cyclooxygenase-2 disrupts tumor vascular mural cell recruitment and survival signaling. Cancer Res. 2006;66:4378-4384.

View Article Google Scholar

15. Huang J, Soffer SZ, Kim ES, McCrudden KW, New T, Manley CA, Middlesworth W, O'Toole K, Yamashiro DJ, Kandel JJ. Vascular remodeling marks tumors that recur during chronic suppression of angiogenesis. Mol Cancer Res. 2004;2:36-42.

View Article Google Scholar

16. Benjamin LE, Hemo I, Keshet E. A plasticity window for blood vessel remodelling is defined by pericyte coverage of the preformed endothelial network and is regulated by PDGF-B and VEGF. Development. 1998;125:1591-1598. View Article Google Scholar

17. Bergers G Song S Meyer-Morse N Bergsland E Hanahan D Benefits of targeting both pericytes and endothelial cells in the tumor vasculature with kinase inhibitors J Clin Invest 200311112871295154450 10.1172/JCI200317929

18. Erber R, Thurnher A, Katsen AD, Groth G, Kerger H, Hammes HP, Menger MD, Ullrich A, Vajkoczy P. Combined inhibition of VEGF and PDGF signaling enforces tumor vessel regression by interfering with pericyte-mediated endothelial cell survival mechanisms. FASEB J. 2004;18:338-340.

View Article Google Scholar

19. Vandesompele J, De Preter K, Pattyn F, Poppe B, Van Roy N, De Paepe A, Speleman F. Accurate normalization of realtime quantitative RT-PCR data by geometric averaging of multiple internal control genes. Genome Biol. 2002;:-.

View Article Google Scholar

20. Team R-DC, (Ed.): R: A language and environment for statistical computing. Vienna: R Foundation for Statistical Computing. 2009

21. Borenstein M, Hedges LV, Higgins JPT, Rothstein HR. An Introduction to Meta-Analysis. Chichester: John Wiley \& Sons Ltd. 2009;:- .

View Article Google Scholar

22. Huang J, Frischer JS, New T, Kim ES, Serur A, Lee A, Kadenhe-Chiwishe A, Pollyea DA, Yokoi A, Holash J, et al. TNP470 promotes initial vascular sprouting in xenograft tumors. Mol Cancer Ther. 2004;3:335-343.

View Article Google Scholar

23. Hulit J, Suyama K, Chung S, Keren R, Agiostratidou G, Shan W, Dong X, Williams TM, Lisanti MP, Knudsen K, Hazan RB. N-cadherin signaling potentiates mammary tumor metastasis via enhanced extracellular signalregulated kinase activation. Cancer Res. 2007;67:3106-3116.

View Article Google Scholar

24. Fayard B, Bianchi F, Dey J, Moreno E, Djaffer S, Hynes NE, Monard D. The serine protease inhibitor protease nexin1 controls mammary cancer metastasis through LRP-1-mediated MMP-9 expression. Cancer Res. 2009;69:56905698.

View Article Google Scholar

25. Larkins TL Nowell M Singh S Sanford GL Inhibition of cyclooxygenase-2 decreases breast cancer cell motility, invasion and matrix metalloproteinase expression BMC Cancer 20066 1811559713 10.1186/14712407-6-181

26. Calvo A Catena R Noble MS Carbott D Gil-Bazo I Gonzalez-Moreno O Huh JI Sharp R Qiu TH Anver MR Identification of VEGF-regulated genes associated with increased lung metastatic potential: functional involvement of tenascin-C in tumor growth and lung metastasis Oncogene 200827537353842702869 10.1038/onc.2008.155

27. Gocheva V, Wang HW, Gadea BB, Shree T, Hunter KE, Garfall AL, Berman T, Joyce JA: IL-4 induces cathepsin protease activity in tumor-associated macrophages to promote cancer growth and invasion. Genes Dev. 24: 241-255.

28. Shreedhar V, Giese T, Sung VW, Ullrich SE. A cytokine cascade including prostaglandin E2, IL-4, and IL-10 is responsible for UV-induced systemic immune suppression. J Immunol. 1998;160:3783-3789.

View Article Google Scholar

29. Huang J, Soffer SZ, Kim ES, McCrudden KW, Huang J, New T, Manley CA, Middlesworth W, O'Toole K, Yamashiro DJ, Kandel JJ. Vascular remodeling marks tumors that recur during chronic suppression of angiogenesis. Mol Cancer Res. 2004;2:36-42.

View Article Google Scholar 
